\title{
Influence of Internal Stresses in Few-Mode Fiber on the Thermal Characteristics of Regenerated Gratings
}

\author{
Nurul Asha MOHD NAZAL ${ }^{1, *}$, Kok Sing LIM ${ }^{1}$, Yen Sian LEE ${ }^{1}$, \\ Muhammad Aizi MAT SALIM ${ }^{2}$, and Harith AHMAD ${ }^{1}$ \\ ${ }^{1}$ Photonics Research Centre, University of Malaya, Kuala Lumpur 50603, Malaysia \\ ${ }^{2}$ Laser Center, Ibnu Sina Institute for Scientific and Industrial Research, Universiti Teknologi Malaysia, Johor Bahru \\ 81310, Malaysia \\ *Corresponding author: Nurul Asha MOHD NAZAL_ E-mail: n.asha2704@yahoo.com
}

\begin{abstract}
The pre-treatment of few-mode fibers (FMFs) has been successfully done with $\mathrm{CO}_{2}$ laser. The wavelength difference, $\Delta \lambda$ between the two resonant wavelengths in the few-mode fiber Bragg grating (FMFBG) varies with temperature increment during the annealing process. The results show that the treated fibers with lower stresses have lower thermal sensitivity in $\Delta \lambda$ than that of non-treated fiber. However, the treated fibers produce FMFBGs with better thermal durability and regeneration ratio. It is conceived that the presence of those stresses in the pristine fiber is responsible for the high thermal sensitivity in $\Delta \lambda$. The thermal relaxation of stresses and structural rearrangement during the thermal annealing process are responsible for the degradation of the strength and resilience of the regenerated grating.
\end{abstract}

Keywords: Regenerated fiber Bragg grating; few-mode fiber; thermal stress relaxation; $\mathrm{CO}_{2}$ laser annealing; thermal resilience

Citation: Nurul Asha MOHD NAZAL, Kok Sing LIM, Yen Sian LEE, Muhammad Aizi MAT SALIM, and Harith AHMAD, "Influence of Internal Stresses in Few-Mode Fiber to the Thermal Characteristics of Regenerated Gratings," Photonic Sensors, 2019, 9(2): 162-169.

\section{Introduction}

Fiber Bragg Grating (FBG) offers an important alternative as a temperature sensor over conventional sensors. Several aspects including the detection range, temperature sensitivity, stability, and reliability are crucial for greater performance of the FBG temperature sensor. Various methods have been reported for instance FBG coated with different materials to improve the temperature sensitivity [1, 2]. Meanwhile, intense studies have been performed to develop the FBG sensor that are robust and reliable in dynamic environment [3]. The study on regenerated grating $(R G)$ has been shown a great development among researchers, and many proposed theories relating to it have been reported. RG what a promising sensor for application in an extreme temperature condition that can withstand temperatures up to $1295{ }^{\circ} \mathrm{C}$ for prolonged durations $[4,5]$. The initial research of $\mathrm{RG}$ was introduced based on the formation of thermally stable chemical composition gratings in optical fiber [6]. In the study, chemical composition grating (CCG) which is based on fluorine-germanium-doped silica fibers is investigated. It is found that the degradation of the grating strength is partly due to the diffusion

Received: 16 July 2018 / Revised: 9 October 2018

(C) The Author(s) 2018. This article is published with open access at Springerlink.com

DOI: $10.1007 / \mathrm{s} 13320-018-0527-4$

Article type: Regular 
properties of dopants in a periodic structure during the thermal annealing process. Other than that, Zhang et al. designed and developed a high-temperature resistance RG sensor by using the hydrogen-loaded germanium-doped fiber Bragg grating [7]. The regeneration of the refractive index modulation in the FBG is induced by the periodic change of molecular water within the fiber core that gives stability at high temperatures. Besides that, an ultra-high temperature $\mathrm{RG}$ in boron-codoped germanosilicate optical fiber using $193 \mathrm{~nm} \mathrm{ArF}$ excimer laser over $1000{ }^{\circ} \mathrm{C}$ was presented by Bandyopadhyay et al. [8]. Yang et al. demonstrated the RG inscribed in new glass composition-based photosensitive fiber with temperature resistance up to $1400^{\circ} \mathrm{C}$ [9]. Our earlier investigation [10] has proven that the pre-treatment process has a profound impact on the performance of the RG in terms of temperature resistant and durability. However, in line with the advancement that has been made, there are still unresolved questions and difficulties in characterization of real mechanism in regeneration process. All of these studies focus only on single mode fiber (SMF) which restricts the exploration of the research to a limited extent.

As such, few-mode fiber (FMF) has attracted many researchers for applications in optical communications and sensors with attractive features than SMF [11-15]. Both SMF and FMF have differences in structural dimensions as well as thermo physical properties of the core and cladding. In the comparison with SMF, the core diameter of FMF is larger [16]. The fiber cross-sectional geometry includes shape and dimension of the core as well as fiber optical properties are well known to influence the axial stress profile of the fibers [17, 18]. In [19], FMFBG with larger core size exhibits higher axial stress profile and relates it to the resonant wavelengths shift in the fiber. FMF is capable of supporting more than one transverse mode in the fiber core, thus, the inscription of Bragg grating in FMF generates more than one Bragg wavelength in the reflection spectrum [20]. These differences contribute advantages to the FMF performance in terms of more mode capacity, flexibility, and discrimination accuracy [15]. Apart from the differences that have been mentioned, step-index FMF comprises the same core-cladding index contrast as well as shares the same dopant (Germanium) concentration in SMF. Hence, the numerical aperture (NA) for these fibers is similar. Therefore, by using step-index FMF, possibly more information on what really happens in the fiber during regeneration could be uncovered.

In this paper, the effort is focused on the investigation of $\mathrm{LP}_{01} \leftrightarrow \mathrm{LP}_{01}$ and $\mathrm{LP}_{01} \leftrightarrow \mathrm{LP}_{11}$ modes in the two-mode step-index fibers, and the differences between the two resonant wavelengths vary with temperature increment during thermal annealing treatment. As comparison to pristine FMF, the pretreatment using $\mathrm{CO}_{2}$ laser on FMF has been performed prior to grating inscription to reduce the internal stresses of the fibers.

\section{Experimental methods}

In this investigation, two-mode step-index fibers (2SF, core diameter: $19 \mu \mathrm{m}, \mathrm{OFS}$ ) have been used. Prior to hydrogen loading and Bragg grating inscription, the fiber is treated with $\mathrm{CO}_{2}$ laser annealing treatment on 1-cm length. Figure 1(a) shows the schematic diagram of the $\mathrm{CO}_{2}$ laser annealing for the fiber pre-treatment. The $\mathrm{CO}_{2}$ laser beam is expanded using two convex lenses and then compressed into 10-cm length with the aid of a cylindrical lens. The fiber is placed vertically within the laser beam region. During the annealing treatment, the laser power is increased at a rate of $1 \mathrm{~W}$ for every $15 \mathrm{~s}$ until the max power of $16 \mathrm{~W}$ is reached with a hold time of 2 hours after the ramping process. Then, the fibers undergo a slow cooling process in which the laser power is reduced to zero at the rate of $\sim 0.1 \mathrm{~W}$ per $15 \mathrm{~s}$, and the equivalent reduction rate for annealing temperature is $\sim 4^{\circ} \mathrm{C}$ per $15 \mathrm{~s}$. This pre-treatment with $\mathrm{CO}_{2}$ laser is intended for reducing the frozen-in stresses, and 
the ensuing slow cooling process is to minimize the thermal stress in the fibers. When the fiber is exposed to $\mathrm{CO}_{2}$ laser, the absorbed laser energy increases the temperature of the fiber glass. When approaching the transition temperature, the viscosity of the fiber glass reduces significantly, enabling the restructuring of the glass molecules and stress relaxation in the fiber. The slow cooling process can prevent the frozen-in stresses to be reintroduced in the fiber and at the same time reducing the transition temperature of the fiber glass which is responsible for the high thermal stress in the fiber. For comparison, several pristine fibers herewith known as non-treated fibers will be employed as controlled specimens in this investigation.

Afterwards, both treated and non-treated 2SFs are soaked in highly pressurized hydrogen gas tube $(13.8 \mathrm{MPa})$ for two weeks to enhance their photosensitivities. In the FBG fabrication, $10 \mathrm{~mm}$ long gratings are on both types of fibers using an ArF excimer laser and a phase mask. To stabilize the spectral properties of the FBGs, the fiber must undergo the dehydrogenation process via a thermal annealing process in an oven at $80^{\circ} \mathrm{C}$ for 12 hours.

Subsequently, the produced FBGs are used as a seed grating (SG) for the thermal regeneration annealing process. The grating is inserted into a tube furnace (LT furnace STF25/150-1600) and isothermal annealing procedure is carried out. The experimental setup for the thermal annealing process is presented in Fig. 1(b). The annealing process is initiated from room temperature, $25^{\circ} \mathrm{C}$ up to $900^{\circ} \mathrm{C}$ with a linear temperature ramping at a rate $9{ }^{\circ} \mathrm{C} / \mathrm{min}$. Both $\mathrm{CO}_{2}$ laser treated and non-treated fibers have undergone similar temperature conditions. After the thermal regeneration annealing process, the hood of the furnace is open to allow faster drop in temperature to prevent further thermal annealing treatment on the RG after the experiment. The RG is left in the tube furnace for a day before the durability test is performed, in which the $\mathrm{RG}$ is annealed from $25^{\circ} \mathrm{C}$ up to $1050^{\circ} \mathrm{C}$ at a ramping rate of $10{ }^{\circ} \mathrm{C} / \mathrm{min}$. The test is conducted based on the same experimental setup as illustrated in Fig. 1(b). The reflection spectrum is recorded throughout the process.
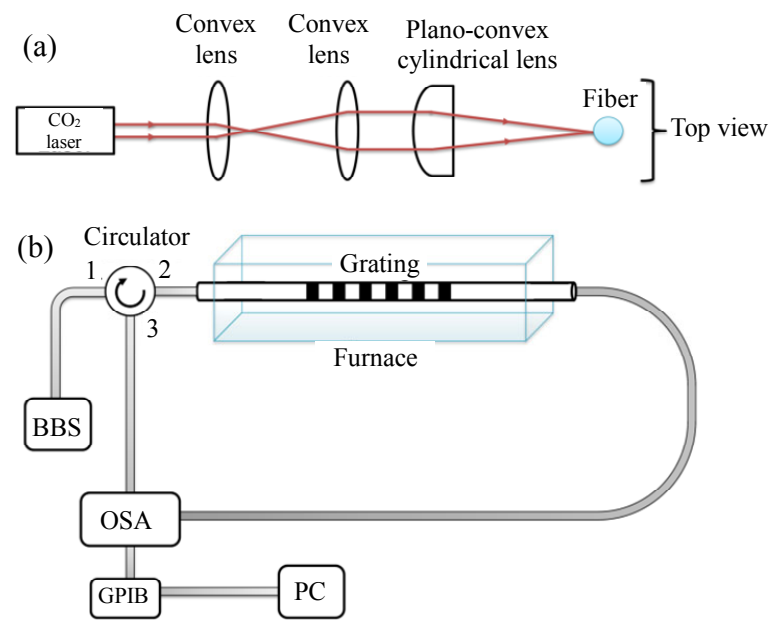

Fig. 1 Schematic diagram of: (a) $\mathrm{CO}_{2}$ laser annealing treatment and (b) fiber thermal annealing using high temperature furnace for regeneration process and durability test. BBS: broadband source, OSA: optical spectrum analyzer, GPIB: general purpose interface bus, and PC: personal computer.

Figure 2 shows the initial transmission spectrum of the grating in $2 \mathrm{SF}$ at room temperature. Since the fiber is fusion spliced to a lead-in SMF, the central axes of both fibers are well aligned to ensure the $2 \mathrm{SF}$ is $\mathrm{LP}_{01}$-excited. This produces two highly reflective resonant wavelengths $\lambda_{a}$ and $\lambda_{b}$ which are associated with $\mathrm{LP}_{01} \leftrightarrow \mathrm{LP}_{01}$ self-mode coupling and $\mathrm{LP}_{01} \leftrightarrow \mathrm{LP}_{11}$ cross mode coupling. On the other hand, the resonant wavelength $\lambda_{c}$ with a low reflectivity can be associated with $\mathrm{LP}_{11} \leftrightarrow \mathrm{LP}_{21}$ cross mode coupling. Since $\mathrm{LP}_{21}$ is a weakly guided mode in this fiber, it will be disregarded in this investigation. In the experiment, the shifts in resonant wavelengths $\lambda_{a}$ and $\lambda_{b}$ are determined from the transmission spectrum. The transmission spectrum is monitored by optical spectrum analyzer (OSA) throughout the whole annealing process and recorded at 1 minute interval using LabVIEW program and a general-purpose interface bus (GPIB) interface card.

Since the dip wavelengths position of the FMFBG is not stable and the peak shape of the wavelengths changes as the temperature increases, it 
is important to use the right peak fitting algorithms. The obtained raw data are post-processed using the center of gravity (COG) method to determine the dip wavelengths. The COG method is a powerful spectral interrogation technique for FBG wavelength with good immunity against the noise and other interference. The estimation is made based on the principle of center of gravity of the reflection curve intensity [21].

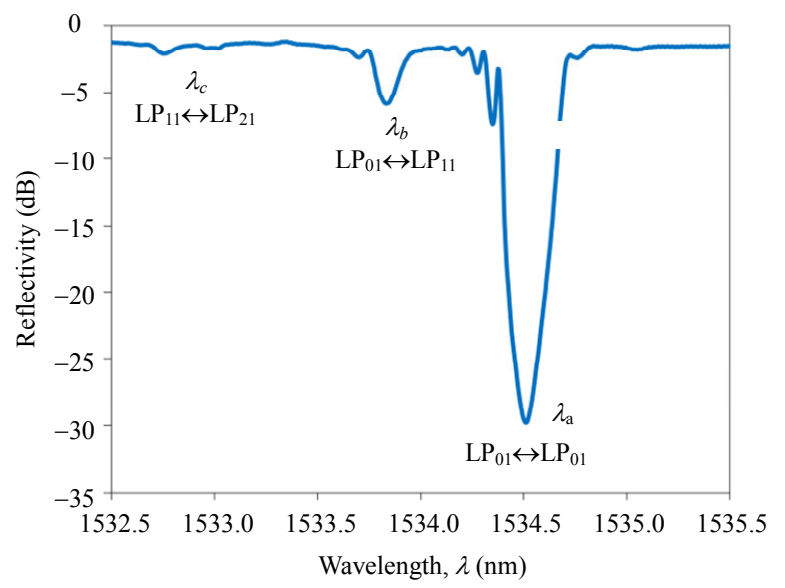

Fig. 2 Transmission spectrum of the $2 \mathrm{SF}$ grating reflectivity before thermal annealing process at room temperature $\left(25^{\circ} \mathrm{C}\right)$.

\section{Results and discussion}

The evolutions of the reflectivity of both non-treated and treated FMFBGs during regeneration process are depicted in Fig. 3. In the figure, the thermal decay and regeneration of the grating reflectivity can be explained by the temporal response of the normalized integrated coupling coefficient (NICC), $\eta$ :

$$
N I C C=\frac{\tanh ^{-1} \sqrt{R_{t, T}}}{\tanh ^{-1} \sqrt{R_{0, T_{0}}}}
$$

where $R_{t, T}$ is the reflectivity after an annealing time $t$ at annealing temperature $T$, and $R_{0, T_{0}}$ is the reflectivity at room temperature before the annealing process.

The annealing temperature is initiated from room temperature $\left(25{ }^{\circ} \mathrm{C}\right)$ until $900{ }^{\circ} \mathrm{C}$ at the ramp rate of $9{ }^{\circ} \mathrm{C} / \mathrm{min}$. In the duration of the first $100 \mathrm{mins}$, $\eta$ decays as the annealing temperature increases linearly from $25{ }^{\circ} \mathrm{C}$ to $900{ }^{\circ} \mathrm{C}$. This is the sign of

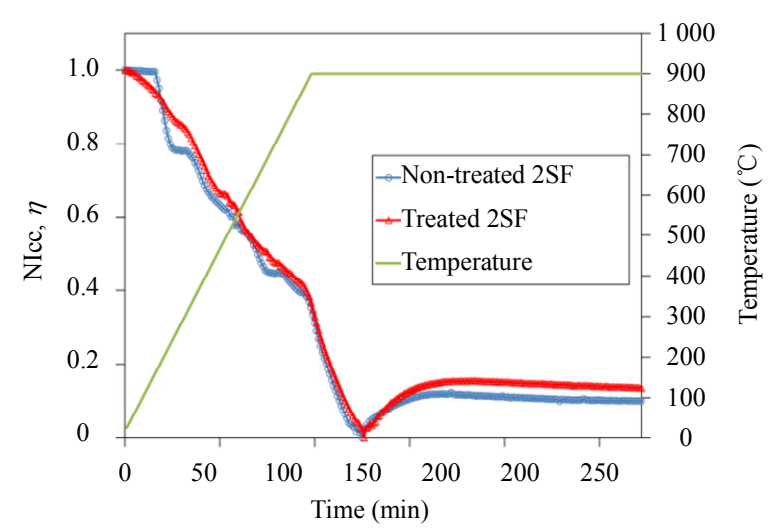

Fig. 3 Reflectivity curves of the non-treated and treated fibers during thermal regeneration process in terms of normalized integrated coupling coefficient (NICC), $\eta$.

thermal decay in UV-induced index change, which is the main constituent to the grating structure. As the annealing temperature is maintained at $900{ }^{\circ} \mathrm{C}, \eta$ continues to decay and becomes 0 at $t=130 \mathrm{~min}$ when the grating is completely erased. Shortly after that, a continuous recovery of the grating is observed as $\eta$ gradually increases and reaches the steady state reflectivity after $t=150$ mins. The significant degradation in reflectivity in the duration of $100 \mathrm{~min}-150 \mathrm{~min}$ can be attributed to the structural rearrangement in the fiber glass, and the grating structure is disrupted during this chaotic period and hence the zero reflectivity is observed at $t=130 \mathrm{~min}$. After the thermal relaxation is completed, the stresses in fiber glass slowly reach the equilibrium state. The grating reflectivity gradually increases, and it reaches the optimum level at $t=150 \mathrm{~min}$. In this work, the efficiency of the regeneration of grating is indicated by the regeneration ratio [10]. The regeneration ratio denotes the ratio of the acquired maximum grating strength of the RG to the initial grating strength of the seed FBG. As observed from the figure, the treated grating regenerates higher reflectivity than non-treated one. The obtained regeneration ratios from the experiment are 0.15 and 0.12 for treated and non-treated fibers, respectively. In the treated fiber, the seed grating is inscribed in the fiber where stress relaxation is already achieved during the pre-treatment process. Therefore, during the 
regeneration process, less deformation in grating structure takes place which contributes to the higher regeneration ratio. On the contrary, for the non-treated fiber, the relaxation of the stresses happens during the regeneration process, which allows more alteration in the grating structure that disturbs the regeneration of the grating. Hence, a lower regeneration ratio occurs. This is affirmed that the stresses that present in the pristine fiber give significant impact on the strength and resilience of the RG.

The transmission and reflection spectra at room temperature of treated and non-treated fibers that display an effect of temperature on the gratings are depicted in Figs. 4(a) and 4(b), respectively. The plots indicate that the wavelengths of RG for both fibers have blue-shifted, and the reflection bandwidths are narrowed down after the thermal regeneration procedure. The shift in the wavelength might be due to the reduction of the DC component in the index change and also the fast cooling after the thermal regeneration [22]. Fast cooling process might reintroduce the thermal stress and small negative-index change in fibers. In Fig. 4(c), the treated fiber exhibits the temperature sensitivities of $14.4 \mathrm{pm} /{ }^{\circ} \mathrm{C}$ for $\lambda_{a}$ and $\lambda_{b}$. Meanwhile, for the non-treated fiber the temperature sensitivities of $14.5 \mathrm{pm} /{ }^{\circ} \mathrm{C}$ for $\lambda_{a}$ and $\lambda_{b}$ are observed as shown in Fig. 4(d). Figures 5 (a) and 5(b) show the relationship between the wavelength difference, $\Delta \lambda$ $\left(\lambda_{a}-\lambda_{b}\right)$, and temperature of the treated fibers and non-treated fibers during the thermal annealing regeneration process. As described by the red best fit lines, both graphs show a linear response in $\Delta \lambda$ with an increase in temperature. The escalation of $\Delta \lambda$ in both fibers suggests that there are some changes in waveguide properties in the fiber during the annealing. The obtained slope value for non-treated fiber is $2.91 \times 10^{-2} \mathrm{pm} /{ }^{\circ} \mathrm{C}$ whereas the slope value for treated fiber is $1.41 \times 10^{-2} \mathrm{pm} /{ }^{\circ} \mathrm{C}$. The grating in non-treated fiber exhibits a larger sensitivity to temperature change compared with the treated ones.

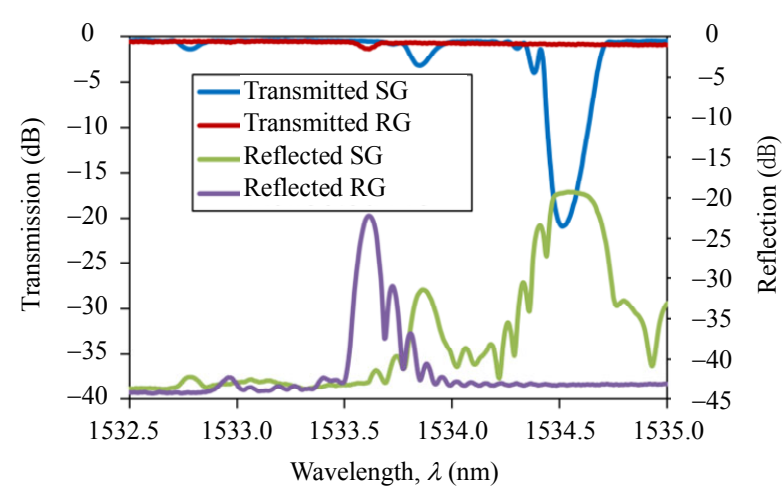

(a)

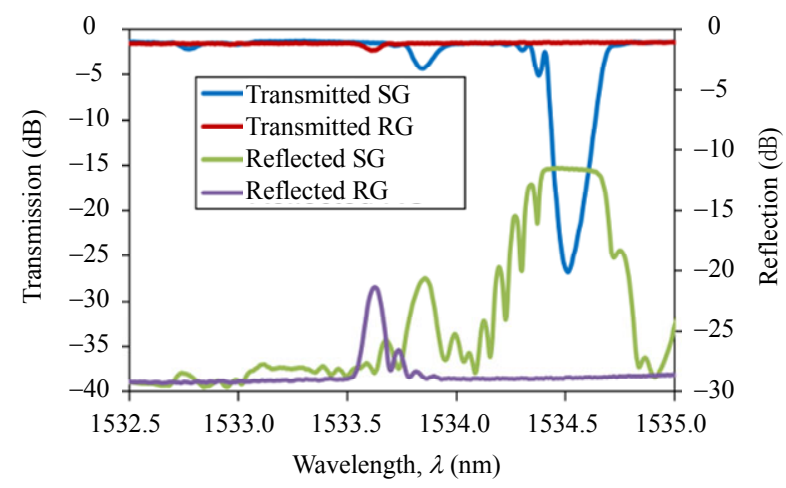

(b)

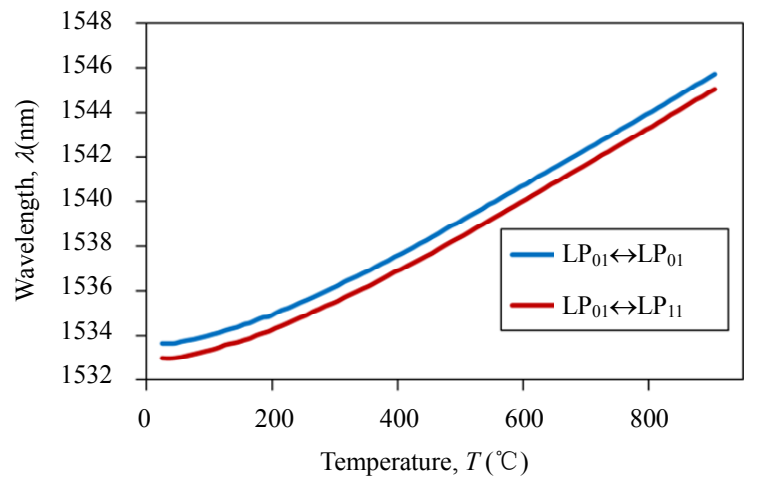

(c)

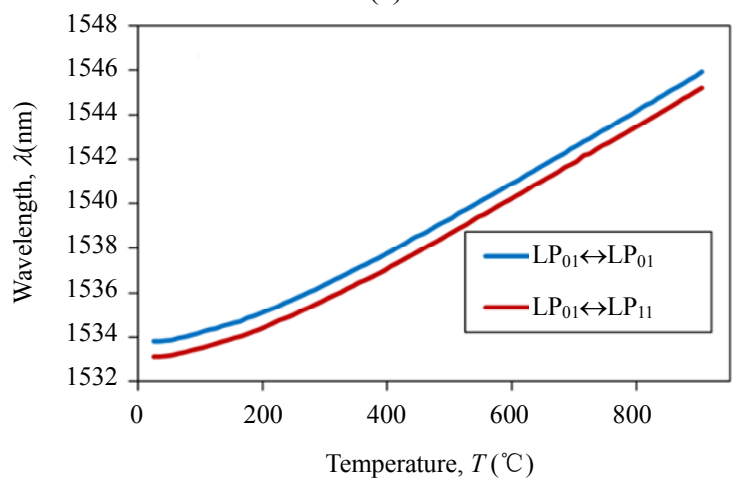

(d)

Fig. 4 Transmission and reflection spectra of SG and RG at room temperature of: (a) treated fiber and (b) non-treated fiber. The wavelength response of $\mathrm{RG}$ in (c) treated fiber and (d) non-treated fiber with the increasing temperature in the range of $25{ }^{\circ} \mathrm{C}-900{ }^{\circ} \mathrm{C}$. 


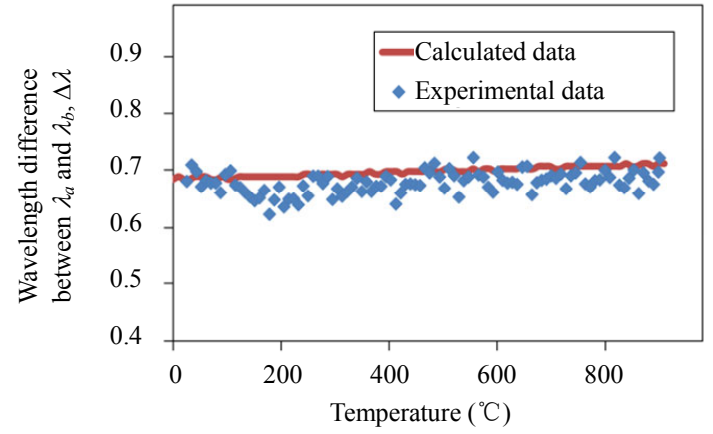

(a)

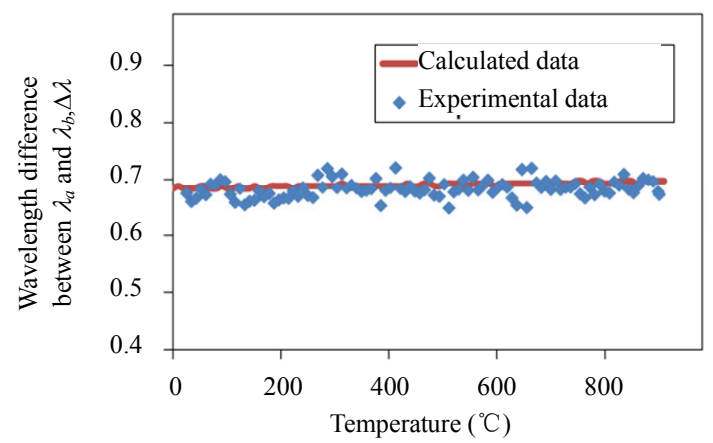

(b)

Fig. 5 Experimental data with linear fit for the wavelength difference between $\lambda_{a}$ and $\lambda_{b}$ as a function of a temperature, $T\left({ }^{\circ} \mathrm{C}\right)$ for (a) non-treated fiber (Fiber 1 ), the slope value is $2.91 \times 10^{-2} \mathrm{pm} /{ }^{\circ} \mathrm{C}$ and (b) $\mathrm{CO}_{2}$ laser-treated fibers (Fiber 4), the slope value is $1.41 \times 10^{-2} \mathrm{pm} /{ }^{\circ} \mathrm{C}$.

We observe the similar trend in another 4 different 2SF specimens, two of which are non-treated fibers (Fibers 2 and 3 ) and the other two are treated fibers (Fibers 5 and 6) as depicted in Fig. 6. The slope values for non-treated fibers (Fibers $1-3$ ) are in the range of $2.5 \times 10^{-2} \mathrm{pm} /{ }^{\circ} \mathrm{C}-$ $3.0 \times 10^{-2} \mathrm{pm} /{ }^{\circ} \mathrm{C}$, whereas the slope values for treated fibers (Fibers 4-6) are in the range of $1.01 .5 \times 10^{-2} \mathrm{pm} /{ }^{\circ} \mathrm{C}-1.5 \times 10^{-2} \mathrm{pm} /{ }^{\circ} \mathrm{C}$. It is believed that the presence of high frozen stresses in non-treated fibers and the thermal relaxation that takes place during the annealing process contributes to a larger wavelength difference $\Delta \lambda$. It is well known that the pristine fiber contains thermal stresses and frozen-in stresses, and the latter are built up during the fiber fabrication process [23, 24]. When the fiber subjects to the high temperature (approaching transition temperature $\left(\sim 1200^{\circ} \mathrm{C}\right)$ [25]), the fiber glass viscosity decreases drastically which allows stress relaxation to take place in the non-treated fiber. The gradual decay in the frozen-in stresses with increasing temperature leads to an increase in resultant material refractive index in the fiber core [10]. In combination with the thermo-optic effect, the fiber glass material refractive index also increases with temperature increment, which explains the higher thermal sensitivity of non-treated fibers as depicted in Fig. 6.

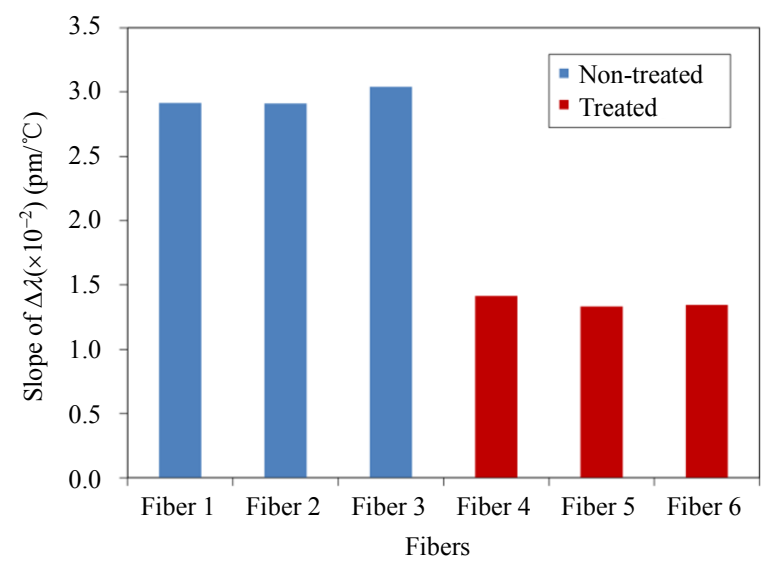

Fig. 6 Comparison of the slope value of $\Delta \lambda$ for the treated fibers and non-treated fibers during thermal regeneration process.

After the first annealing process, the fibers are left in the furnace for a day and go through a slow cooling process to room temperature to ensure that the minimum thermal stresses are built up in the fiber since the thermal annealing cannot completely eliminate the thermal stress within the fiber. Then, the fibers are again subjected to another thermal annealing process for 10 hours up to $1050{ }^{\circ} \mathrm{C}$. Figure 7 shows the variation of the slope of $\Delta \lambda$ in both treated and non-treated fibers in the post annealing process. It can be observed that the slope values of $\Delta \lambda$ for all of the fibers are much lower after the post thermal annealing except Fiber 3 because the reflectivity of the resonant wavelengths in Fiber 3 is too low and it cannot be determined. The entire fibers exclude Fiber 6 exhibit the slope values in the range from $0.1 \times 10^{-2} \mathrm{pm} /{ }^{\circ} \mathrm{C}$ to $0.5 \times 10^{-2} \mathrm{pm} /{ }^{\circ} \mathrm{C}$. This indicates that less stress relaxation and structural rearrangement are resulted 
during the post annealing as all the fibers are almost attained to a steady state in terms of mechanical stress and structural stability. However, the change in the wavelength difference still happens during the process, and it is believed that the residue frozen-in stresses in the fibers are responsible for this behavior. The thermal decay rate for the $R G$ in treated fiber is $0.805 \times 10^{-2} \mathrm{~dB} / \mathrm{min}$ whereas the decay rate for non-treated fiber is $1.24 \times 10^{-2} \mathrm{~dB} / \mathrm{min}$. Again, this is consistent with our hypothesis that frozen-in stresses are responsible for degradation of grating structure and reflectivity. The absence of these stresses in the treated fibers provides a more thermally stable host for the gratings, making them more resilient in a high temperature environment.

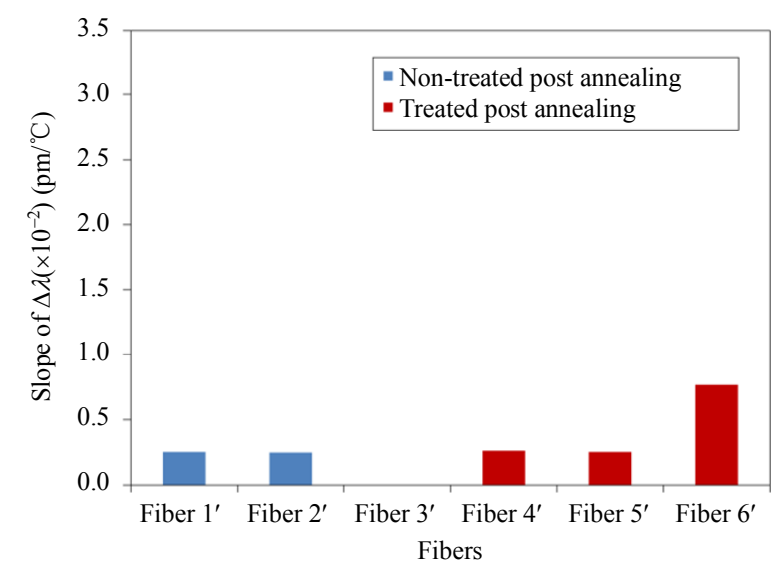

Fig. 7 Slope value $\Delta \lambda$ of wavelength difference between $\lambda_{a}$ and $\lambda_{b}$ for treated and non-treated fibers during post annealing process (durability test).

\section{Conclusions}

In this paper, the thermal annealing of pre-treatment two-mode FBG is demonstrated. In order to determine the position of the wavelengths dip precisely and accurately, the COG method is applied to characterize the obtained data. This approach can ease the analysis and prediction of small wavelength shift with the temperature change. From the experiment, we have observed a linear correlation between wavelength difference, $\Delta \lambda$ and temperature. The analysis also indicates that the thermal sensitivity of $\Delta \lambda$ for treated FMFs is lower than that of non-treated one, which suggests that the presence of frozen-in stresses and high thermal stresses are responsible for the higher thermal sensitivity of $\Delta \lambda$. The treated fibers with lower stresses produce RGs with better regeneration ratio and thermal durability. This shows that pre-treatment process offers stability in term of less structural arrangement and makes the grating more durable. The thermal sensitivity of $\Delta \lambda$ can be used as an indicator for the stresses condition in the fibers. The results from this work provide a new insight in the exploration of the real mechanisms behind $\mathrm{RG}$ and the influence of stresses in the fibers to the performance of RGs.

\section{Acknowledgement}

This work was performed within an Optical Fibers Sensors and Lasers Laboratory. The first author acknowledges all postgraduates students from the lab for general support. This work was supported by the Fundamental Research Grant Scheme (FRGS) (Grant No. FP033-2017A).

Open Access This article is distributed under the terms of the Creative Commons Attribution 4.0 International License (http://creativecommons.org/licenses/by/4.0/), which permits unrestricted use, distribution, and reproduction in any medium, provided you give appropriate credit to the original author(s) and the source, provide a link to the Creative Commons license, and indicate if changes were made.

\section{References}

[1] T. C. Hsiao, T. S. Hsieh, Y. C. Chen, S. C. Huang, and C. C. Chiang, "Metal-coated fiber Bragg grating for dynamic temperature sensor," Optik, 2016, 127(22): 10740-10745.

[2] Y. L. Li, K. Yang, and X. W. Li, "Temperature sensing characteristics of metal coated FBG during dynamic cooling process," Optical Fiber Technology, 2018, 45: 368-375.

[3] D. S. Gunawardena, M. H. Lai, K. S. Lim, A. Malekmohammadi, and H. Ahmad, "Fabrication of thermal enduring FBG sensor based on thermal induced reversible effect," Sensors and Actuators A: Physical, 2016, 242: 111-115.

[4] J. Canning, M. Stevenson, S. Bandyopadhyay, and K. 
Cook, "Extreme silica optical fibre gratings," Sensors, 2008, 8(10): 6448-6452.

[5] S. J. Mihailov, "Fiber Bragg grating sensors for harsh environments," Sensors, 2012, 12(2): 1898-1918.

[6] M. Fokine, "Formation of thermally stable chemical composition gratings in optical fibers," Journal of the Optical Society of America B, 2002, 19(8): 1759-1765.

[7] B. Zhang and M. Kahrizi, "High-temperature resistance fiber Bragg grating," IEEE Sensors Journal, 2007, 7(4): 586-591.

[8] S. Bandyopadhyay, J. Canning, M. Stevenson, and K. Cook, "Ultrahigh-temperature regenerated gratings in boron-codoped germanosilicate optical fiber using 193 nm," Optics Letters, 2008, 33(16): 1917-1919.

[9] H. Z. Yang, X. G. Qiao, S. Das, and M. C. Paul, "Thermal regenerated grating operation at temperatures up to $1400{ }^{\circ} \mathrm{C}$ using new class of multimaterial glass-based photosensitive fiber," Optics Letters, 2014, 39(22): 6438-6441.

[10] N. A. M. Nazal, K. S. Lim, M. K. A. Zaini, H. Z. Yang, and H. Ahmad, "Formation of enhanced regenerated grating in few-mode fiber by $\mathrm{CO}_{2}$ laser pretreatment," Applied Optics, 2017, 56(36): 9882-9887.

[11] A. Li, A. Al Amin, X. Chen, and W. Shieh, "Transmission of $107 \mathrm{~Gb} / \mathrm{s}$ mode and polarization multiplexed CO-OFDM signal over a two-mode fiber," Optics Express, 2011, 19(9): 8808-8814.

[12] A. Li, A. Al Amin, X. Chen, S. Chen, G. Gao, and W. Shieh, "Reception of dual-spatial-mode CO-OFDM signal over a two-mode fiber," Journal of Lightwave Technology, 2012, 30(4): 634-640.

[13] C. Koebele, M. Salsi, D. Sperti, P. Tran, P. Brindel, H. Mardoyan, et al., "Two mode transmission at $2 \times 100 \mathrm{~Gb} / \mathrm{s}$, over $40 \mathrm{~km}$-long prototype few-mode fiber, using LCOS-based programmable mode multiplexer and demultiplexer," Optics Express, 2011, 19(17): 16593-16600.

[14] B. Y. Kim, "Few-mode fiber devices," Optical Fiber Sensors, 1988, 2: 1-463.

[15] A. Li, Y. Wang, Q. Hu, and W. Shieh, "Few-mode fiber based optical sensors," Optics Express, 2015, 23(2): 1139-1150.
[16] N. A. M. Nazal, M. H. Lai, K. S. Lim, D. S. Gunawardena, W. Y. Chong, H. Z. Yang, et al., "Demarcation energy properties of regenerated fiber Bragg grating sensors in few-mode fibers," Optics Applicata, 2018, 48(2): 263-271.

[17] P. Chu and R. Sammut, "Analytical method for calculation of stresses and material birefringence in polarization-maintaining optical fiber," Journal of Lightwave Technology, 1984, 2(5): 650-662.

[18]K. S. Lim, Y. H. Zhou, W. Y. Chong, C. Y. Ken, C. H. Lim, N. M. Ali, et al., "Axial contraction in etched optical fiber due to internal stress reduction," Optics Express, 2013, 21(3): 2551-2562.

[19] M. K. A. Zaini, Y. S. Lee, K. S. Lim, N. A. M. Nazal, M. H. Zohari, and H. Ahmad, "Axial stress profiling for few-mode fiber Bragg grating based on resonant wavelength shifts during etching process," Journal of the Optical Society of America B, 2017, 34(9): 1894-1898.

[20] T. Mizunami, T. V. Djambova, T. Niiho, and S. Gupta, "Bragg gratings in multimode and few-mode optical fibers," Journal of Lightwave Technology, 2000, 18(2): 230-235.

[21]D. Ganziy, O. Jespersen, G. Woyessa, B. Rose, and O. Bang, "Dynamic gate algorithm for multimode fiber Bragg grating sensor systems," Applied Optics, 2015, 54(18): 5657-5661.

[22] M. H. Lai, K. S. Lim, D. S. Gunawardena, and H. Z. Yang, "Thermal stress modification in regenerated fiber Bragg grating via manipulation of glass transition temperature based on $\mathrm{CO} 2$-laser annealing," Optics Letters, 2015, 40(5): 748-751.

[23] I. H. Shin, B. H. Kim, S. P. Veetil, W. T. Han, and D. Y. Kim, "Residual stress relaxation in cleaved fibers," Optics Communications, 2008, 281(1): 75-79.

[24] B. H. Kim, Y. Park, T. J. Ahn, D. Kim, B. H. Lee, Y. Chung, et al., "Residual stress relaxation in the core of optical fiber by $\mathrm{CO}_{2}$ laser irradiation," Optics Letters, 2001, 26(21): 1657-1659.

[25] J. A. Bucaro and H. D. Dardy, "High-temperature Brillouin scattering in fused quartz," Journal of Applied Physics, 1974, 45(12): 5324-5329. 